\title{
Preganglionic Autonomic Motor Neurons Display Normal Translocation Patterns in Slice Cultures of Embryonic Rat Spinal Cord
}

\author{
Robert P. Barber, Patricia E. Phelps, and James E. Vaughn \\ Division of Neurosciences, Beckman Research Institute of the City of Hope, Duarte, California 91010
}

Phenotypic differences between somatic and autonomic motor neurons (SMNs and AMNs, respectively) may be modulated by epigenetic factors during the histogenic migrations of these cells. In order to study this problem experimentally, we have developed an in vitro, organotypic slice preparation of embryonic rat spinal cord. Our main objectives for this preparation were to determine whether in vivo patterns of motor neuronal translocations were mimicked in vitro, and, if they were, to begin to analyze such movements with experimental procedures that cannot be applied to the study of mammalian spinal cord development in vivo. Using a modification of existing organotypic slice procedures, we have shown that ChAT, an axonal surface glycoprotein and a lowmolecular-weight neurofilament protein are expressed in slices cultured for up to $21 \mathrm{~d}$, thus indicating that spinal neurons remained viable in vitro for relatively long periods. Most importantly, retrograde labeling and subsequent confocal microscopy have shown that the SMNs and AMNs of the slice preparations become segregated ventrodorsally into two distinct subcolumns as seen in vivo. The formation of separate AMN and SMN subcolumns appears to result from a dorsal translocation of AMNs. The fact that this cellular movement occurs in the slice preparation has allowed us to follow this phenomenon directly within the same specimen over a period of days. In addition, we have been able to observe the translocation of AMNs following the removal of their peripheral synaptic targets. The results of these experiments provide further evidence that AMNs undergo a dorsal translocation during the course of spinal cord development, and that this cellular movement may be due to an active migration. They also indicate that $A M N$ movement is not dependent upon continual connection of these neurons with the paravertebral sympathetic ganglia.

\footnotetext{
Received Feb. 16, 1993; revised May 10, 1993; accepted May 24, 1993.

We thank Drs. Garrett Crawford and Paul Salvaterra (anti-ChAT antibody, 3F12), Dr. Miyuki Yamamoto (anti-SNAP/TAG-1 antibody, 4D7), Dr. Arlene Chiu (anti-neurofilament antibody, MO-3d), and Dr. Marcia Miller (control antibody against chick erythrocyte membrane) for generously providing immunoreagents used in the present investigation. In addition, we are grateful to our colleagues, Drs. Arlene Chiu, Lisa Dahm, and Rick Wetts, for their constructive criticisms and comments regarding this article. We also acknowledge the expert technical assistance of Mariko Lee, Christine Vaughn, and Lynn Brennan, as well as the sceretarial expertise of Marilyn Ashley. This work was supported by NIH Grant NS25784.

Correspondence should be addressed to Dr. James E. Vaughn, Division of Neurosciences, Beckman Research Institute of the City of Hope, 1450 East Duarte Road, Duarte, CA 91010.
}

Copyright (C) 1993 Society for Neuroscience $0270-6474 / 93 / 134898-10 \$ 05.00 / 0$
[Key words: nervous system, development, migration, phenotypic differentiation, cholinergic neurons, SNAP/TAG-1, confocal microscopy, D:I labeling, synaptic target removal]

Discovering how cellular diversity is generated during neuronal differentiation is an important challenge facing developmental neurobiologists. We have chosen to explore one aspect of the differentiation of neuronal phenotypes by studying two closely related subsets of spinal cord motor neurons: the somatic motor neurons (SMNs) that innervate striated muscle, and the autonomic motor neurons (AMNs or preganglionic neurons) that innervate the peripheral neurons of the sympathetic nervous system. In previous studies, evidence has been provided that these two motor neuronal subsets (1) have the same birthdays (Barber et al., 1991) and, possibly, a similar site of origin within the ventral ventricular zone (Phelps et al., 1991a); (2) are probably clonally related to one another (Leber et al., 1990); (3) leave the ventricular zone together to form a common, primitive motor column (Phelps et al., 1991a); and (4) begin to express their neurotransmitter synthesizing enzyme, ChAT, about the time that they form the primitive motor column (Phelps et al., 1991a). Furthermore, both subsets of motor neurons initially are intermixed in this common column before AMNs become segregated near its dorsal pole while SMNs remain located ventrally (Markham and Vaughn, 1991). Following this dorsoventral segregation, AMNs exhibit secondary, progressive translocations cither dorsally or dorsomedially until they attain their characteristic spinal locations just prior to birth (Markham and Vaughn, 1991; Phelps et al., 1991a, 1993). Although SMNs and AMNs share a number of common characteristics as outlined above, they also exhibit phenotypic differences in such features as size, spinal location, dendritic patterns, and specific synaptic targets in the periphery. While the precise factors mediating these differences are unknown, the various migratory routes taken by these two subsets of spinal motor neurons may express epigenetic signals that trigger the differentiation of these CNS cells in much the same way as diverse migration pathways are thought to play a role in the differentiation of neural crest derivatives in the periphery (Bronner-Fraser and Fraser, 1988).

Developmental patterns of motor neuronal migration have been inferred from observations obtained with the independent techniques of ChAT immunocytochemistry (Phelps et al., 1991a) and HRP retrograde labeling (Markham and Vaughn, 1991). Although the descriptive in vivo results from these two methods agree well with one another, they do not provide a direct demonstration of cellular migrations. Such a demonstration is difficult in mammalian embryos because they are relatively inaccessible to experimental analyses. In an attempt to obviate 
this difficulty, we have developed an in vitro slice preparation of embryonic rat spinal cord. This preparation allows direct observation of cellular movements during development, as well as experimental manipulations designed to reveal the mechanisms of ontogenetic events. We have determined that the embryonic spinal cord maintains its essential histotypical features in slice culture. Furthermore, it has been possible to observe motor neuronal translocations directly in the slices and to determine that they mimicked those inferred in vivo. Moreover, it has been found that AMN translocations in the in vitro slice can occur without continuous contact between these cells and their synaptic targets in the periphery.

\section{Materials and Methods}

\section{Experimental animals and embryo preparations}

Adult, female Sprague-Dawley rats were placed in breeding cages overnight with males. Vaginal smears were prepared the following day to verify insemination, and the day that a positive smear was observed was designated as embryonic day 1 (E1). This method of staging embryos makes specimens used in this study appear to be a day older than those of investigators who designate the day following insemination as E0. Pregnant rats were deeply anesthetized with $2-2.5 \%$ halothane administered with a precision vaporizer. Embryos (E14 and E16) were removed individually by cesarean section with their placentas and embryonic membranes intact, and placed into sterile, cold Gey's balanced salt solution (Freshney, 1987) that was gassed continuously with a mixture of $5 \% \mathrm{CO}_{2}$ and $95 \% \mathrm{O}_{2}$.

Prior to sectioning, the placentas and embryonic membranes were dissected, and the head and developing viscera were surgically removed from each embryo. The resulting trunk segment and attached forelimbs were embedded in $7.3 \%$ agarose (Sigma, type IX ultralow gelation temperature). These trunk segments included the embryonic spinal column containing the spinal cord and associated dorsal root ganglia, part of the aorta, and the paravertebral chain of sympathetic ganglia, as well as developing somatic musculature in both the trunk and attached forelimbs.

\section{Spinal cord slices}

Agarose blocks were trimmed and mounted for transverse sectioning of embryonic trunk segments with an Oxford vibratome. Sections, 300$400 \mu \mathrm{m}$ thick, were placed in sterile, cold Gey's solution where the surrounding agarose was removed before slices were transferred by spatula to a drop of chicken plasma (Sigma, P-3266) on the bottom of a culture dish. A drop of thrombin (Sigma, T-6634; $250 \mathrm{U}$ ) was added to each specimen, and a clot formed that anchored the spinal slice to the culture dish. Alteratively, slices were affixed to the culture dish with Cell-Tak (BioPolymers, Inc.). Each culture dish contained two spinal slices, and was filled with $1.5 \mathrm{ml}$ of culture medium consisting of $50 \%$ Dulbecco's Modified Eagle's Medium, 25\% Hank's balanced salt solution, $10 \%$ heat-inactivated fetal calf serum, $15 \%$ distilled water, $0.03 \%$ glutaminc, $0.002 \% \mathrm{H}_{2} \mathrm{O}_{2}$, and $20 \mathrm{ng} / \mathrm{ml}$ nerve growth factor. Up to this point, our procedures for spinal slices were adapted from those of Gähwiler (1981), Gähwiler et al. (1990), and Braschler et al. (1989). Following immersion in culture medium, specimens were placed in a moist, $5 \% \mathrm{CO}_{2}$ incubator at $35.5^{\circ} \mathrm{C}$. The specimens remained stationary for the first $2 \mathrm{hr}$ in culture; thereafter they were gently rocked side to side once every minute so that the slices were completely immersed in medium for only about one-half of the total incubation period. The culture medium was exchanged every $3 \mathrm{~d}$.

\section{Experimental paradigms}

Survival in culture. In order to determine the viability of the embryonic slices, E14 and E16 specimens were cultured for 2-21 d. Viability of these specimens was determined by morphological preservation and the immunocytochemical labeling of a number of molecular markers including choline acetyltransferase (ChAT; antibody $=3 \mathrm{~F} 12$; for methods, see Phelps et al., 1991a), stage-specific neurite associated protein/transiently expressed axonal surface glycoprotein (SNAP/TAG-1; antibody=4D7; Yamamoto et al., 1986; Dodd et al., 1988; for methods, see Vaughn et al., 1992), and low-molecular-weight neurofilament protein (antibody=MO-3d; for details, see Chen and Chiu, 1992).

$A M N$ translocation. Embryonic day 14 specimens were selected for these experiments because both subsets of motor neurons (SMNs and AMNs) were still located within a single, primitive column at this stage of development. Retrograde labeling of motor neurons was accomplished in these experiments by injecting the fluorescent carbocyanine dye Dil (Molecular Probes, Inc.; see Honig and Hume, 1989). Solutions of $0.1 \%$ or $0.5 \%$ DiI, dissolved in either absolute ethanol, dimethylformamide, or vegetable oil (Wesson), were injected from glass micropipettes (tip diameters of $5-10 \mu \mathrm{m}$ ) by air pressure that was regulated with a Picospritzer II (General Valve Co.). Two different injection modes were used. The first method of retrograde labeling (living mode) was to inject $\mathrm{DiI}$ into the ventral roots and paravertebral sympathetic ganglia of the $300-400-\mu \mathrm{m}$-thick spinal slices after a 1-2 hr incubation, during which time the slices became anchored to the culture dish. A change of medium was made subsequent to these injections before the specimens were returned to the incubator for various times in culture. The slices

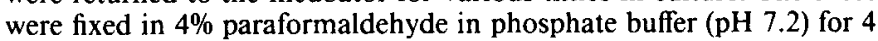
$\mathrm{hr}$ at $4^{\circ} \mathrm{C}$ at the termination of the in vitro period. The second mode for labeling molor neurons (fixed mode) was to culture the slices as described above for various times, fix the slices in $4 \%$ paraformaldehyde, and inject DiI into the same locations as described for the living mode. The fixed injection mode was used to optimize morphological details, and the living injection mode was used for experiments that required labeling at the beginning of the culture period. No significant differences were observed between the results obtained by these two different modes of injection.

The translocation patterns of AMNs in vitro were studied by Dil injections of both fixed and living slices. For the fixed slice injections, slices were obtained at E14 (common motor column stage), and they were cultured for $2,24,48$, or $72 \mathrm{hr}$ before the culture medium was replaced with $4 \%$ paraformaldehyde in Millonig's phosphate buffer. Following fixation for at least $4 \mathrm{hr}$, DiI was injected as described above and these specimens were stored in the dark at $4^{\circ} \mathrm{C}$ for periods up to 6 weeks to allow for retrograde labeling of the AMNs and SMNs. For the living slice injections, the ventral roots and paravertebral ganglia were injected with Dil immediately after the slices became anchored to the culture dishes. Clusters of DiI labeled cells in living spinal slices were followed on a day-to-day basis over a $3 \mathrm{~d}$ period $(24,48$, and $72 \mathrm{hr}$ ) with confocal microscopy, using low magnifications and relatively weak laser intensities to record daily cell movements. Increasing magnification and laser intensity killed DiI-labeled cells, thereby limiting the usefulness of this paradigm to low-resolution images. In order to obtain higher-resolution images of motor neuronal translocation patterns in injected living specimens, E14 spinal slices were injected with DiI, and these slices were fixed either 2 or $72 \mathrm{hr}$ following injection, times known from our previous observations to represent the single and separated motor subcolumn states, respectively.

Ablation of paravertebral ganglia in vitro. Slices of E14 spinal cord were injected with Dil and cultured for $4 \mathrm{hr}$ as detailed above. Specimens were taken from the incubator, and all tissues ventral to the spinal cords, including the paravertebral ganglia, were excised microsurgically and discarded. After $3 \mathrm{~d}$ of culture, the slices were fixed (see above) for confocal microscopy.

\section{Confocal microscopy}

A Zeiss LSM 10 laser scanning confocal microscope was used to observe and record digital images of optical sections $2.5-10 \mu \mathrm{m}$ thick through the entire cultured slice. Digital images were stored on optical disks for subsequent three-dimensional reconstruction and other image analyses with a PC workstation using MiCROvoxel software (INDEC Systems, Inc.; Capitola, CA).

\section{Results}

\section{Characteristics of spinal slices in culture}

Preliminary studies were conducted to determine embryonic spinal slice survivability in culture. Among the criteria essential for subsequent experiments were the survival of both subsets of motor neurons and their connectivity with peripheral synaptic targets. Expression of ChAT was used to demonstrate motor neuronal survival, and the presence of neurofilament protein was used to reveal axonal projections to peripheral targets. In addition, immunocytochemical detection of a surface component of early forming circumferential neurons was used 


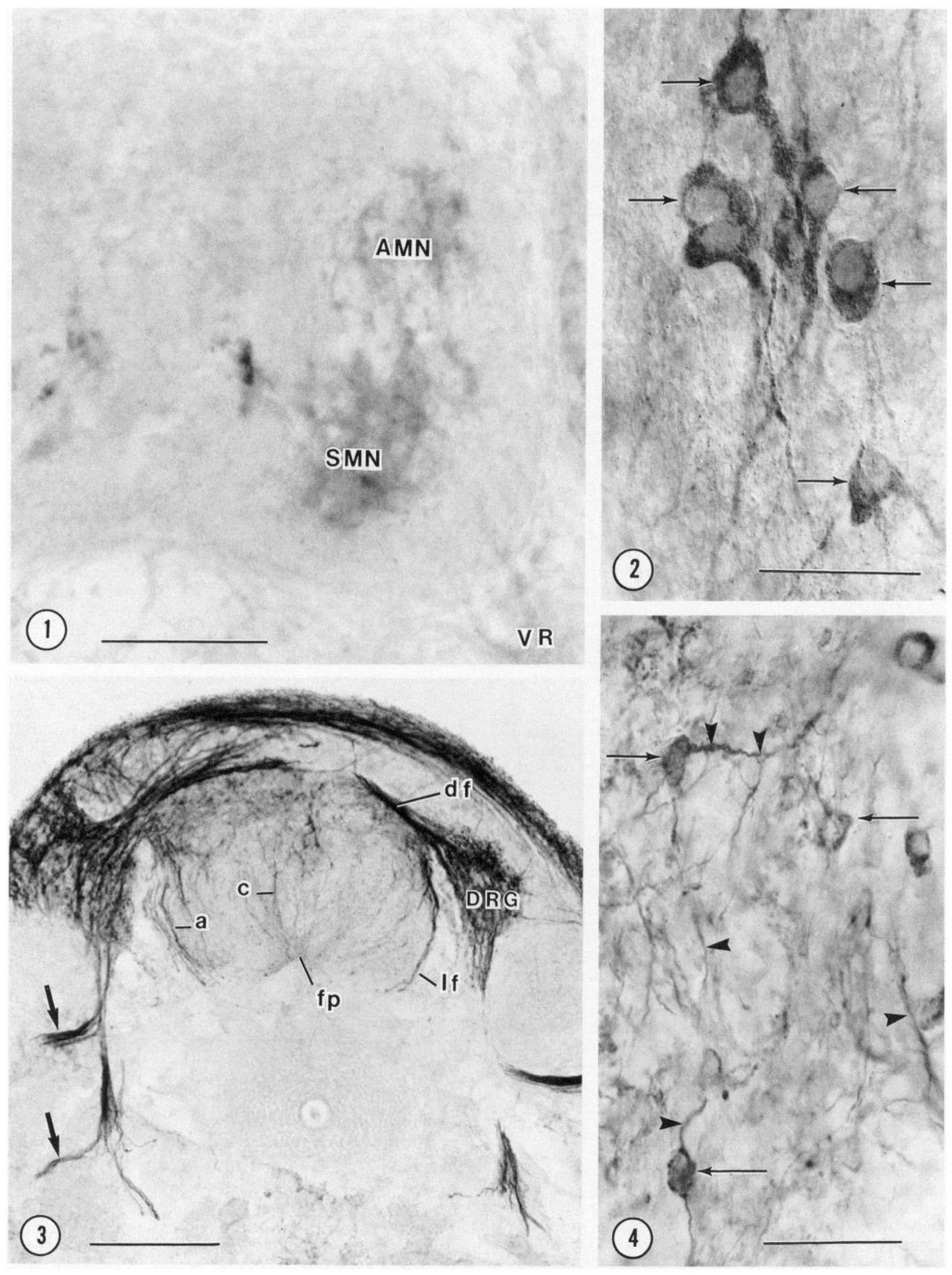


to determinc whether axonal pathways putatively associated with AMN translocation survived in the slice preparations. Slices from E14 and E16 embryos were cultured for periods ranging from 2 to $21 \mathrm{~d}$. Results from these experiments were as follows. First, ChAT was expressed in both AMNs and SMNs of E14 spinal slices cultured for $24 \mathrm{hr}$ (Fig. 1), as well as in ventral horn neurons of E16 slices cultured for 21 d (504 hr, Fig. 2). Second, spinal slices from E14 specimens contained neurons that expressed SNAP/TAG-1 after $2 \mathrm{~d}$ in culture, and a relatively normal appearance was observed for commissural, association, and dorsal root fibers in these specimens (Fig. 3). Third, neurofilament protein was expressed in spinal neurons of E16 slices cultured for up to $96 \mathrm{hr}$ (Fig. 4). Spinal cord slices maintained their characteristic shapes, and exhibited fiber projection into both somatic muscles and the paravertebral sympathetic ganglia (Fig. 5). Such histotypic features were exhibited by many spinal slices for at least $72 \mathrm{hr}$ in culture. However, there were abnormal fiber pathways crossing in both the dorsal part of the spinal slice and between the autonomic ganglia on either side of the midline (Fig. 5). The aberrant pathway in the dorsal spinal cord was composed of dorsal root ganglion fibers, at least in part, and these fibers appeared to be restricted to the upper, outside surface of the slice and did not cross the midline within the parenchyma of the slice proper. Consequently, this relatively minor abnormality had no apparent affect on AMN translocations as determined in the experiments described below.

\section{AMN translocation patterns in vitro}

In addition to the survival and histotypic characteristics described above, the AMN translocation patterns in cultured slices mimicked those observed in vivo. Spinal slices from E14 embryos that were cultured for 2 or 24 hr contained a single column of $A M N s$ and SMNs, as identified by DiI injections into both living (Fig. 6) and fixed (Fig. 7) slices. The dense clustering of motor neurons into a single column at $2 \mathrm{hr}$ in culture throughout the entire slice was confirmed by optical sectioning with the confocal microscope and subsequent three-dimensional reconstructions of the electronically stored images (Fig. 8). In contrast, confocal microscopy of E14 spinal slices cultured for $72 \mathrm{hr}$ revealed that DiI-labeled motor neurons had separated into $t w o$, distinct subcolumns, one located in the ventral horn and the other in the intermediolateral horn (Fig. 9). Optical sectioning and three-dimensional reconstructions confirmed the physical separation between the two subcolumns throughout the entire $\mathrm{z}$-axis of the slices (Fig. 10). In addition to the dorsoventral separation of the two motor subcolumns, some E14 slices cultured for $72 \mathrm{hr}$ contained DiI-labeled neurons that were oriented mediolaterally and appeared to be separating medially from the dorsal, intermediolateral column toward the ventricular zone (Fig. 1 $1 a, b$ ). Relatively normal translocations of AMNs were observed in about three-quarters of the samples, whereas the remaining one-quarter exhibited either slight or no apparent signs of AMN translocation.

Results from E14 spinal slices cultured for $48 \mathrm{hr}$ exhibited stages in the arrangements of motor neurons that were intermediate or transitional between those observed after 24 and 72 $\mathrm{hr}$ in culture. Furthermore, images from the same E14 slices observed on sequential days of culture revcaled a gradual, progressive transition between the single and separated subcolumns of motor neurons (Figs. 12-14). During transitional stages, some neurons were observed that exhibited a relatively simple bipolar orientation in the dorsoventral axis (Fig. 15). In contrast, toward the end of the transition period, some dorsally located neurons were displaced toward the spinal midline and exhibited bipolar neurites oriented mediolaterally (Figs. 15, 16).

\section{Removal of AMN peripheral target tissue}

The following experiments were conducted to determine whether or not AMN connections with their peripheral synaptic targets play a role in the translocation patterns observed for these neurons. Four hours after DiI was injected into the paravertebral ganglia, the ventral parts of embryonic trunk slices containing these ganglia were removed microsurgically. These experimentally altcred preparations were cultured for a total in vitro time of $72 \mathrm{hr}$. Following incubation, the specimens were fixed and examined to determine if the dorsal translocation of AMNs occurred when their normal synaptic targets were no longer present. These samples revealed the same dorsal translocation of AMNs as observed in intact spinal slices that were connected to the paravertebral ganglia. Over one-half of the surgically perturbed samples exhibited a pronounced dorsoventral separation of DiI-labeled cells into two distinct motor subcolumns (Fig. 17), and, in a few cases, evidence of a mediolateral translocation of some of the dorsalmost cells was also apparent (not shown). In another one-quarter of the experimentally altered specimens, there was a dorsal translocation of labeled cells, but no clear separation into distinct subcolumns. This same pattern was also observed occasionally in intact slices. As was the case for intact slices, the remaining one-quarter of the experimentally altcred samples displayed little or no sign of AMN translocation.

\section{Discussion}

Principal findings of this investigation were that the cultured spinal slices remained viable and exhibited histotypic characteristics such as shape and connectivities over the period of time necessary for them to be useful for AMN translocation studies. Most importantly, it was found that the AMN patterns of movement in cultured embryonic spinal slice preparations were very similar to those observed in vivo (Markham and Vaughn, 1991; Phelps et al., 1991a). Furthermore, when the maturation of the

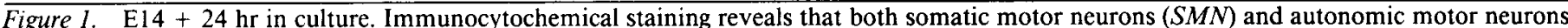

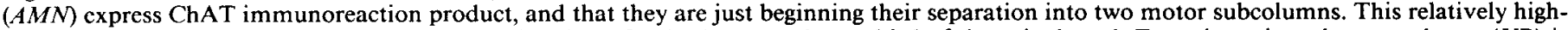

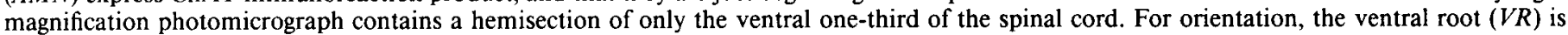
indicated in the lower right corner. Scale bar, $100 \mu \mathrm{m}$.

Figure 2. E16 + $504 \mathrm{hr}$ in culture. Ventral horn neurons (arrows) in a spinal cord slice exhibit the epitope for ChAT. Scale bar, $50 \mu \mathrm{m}$.

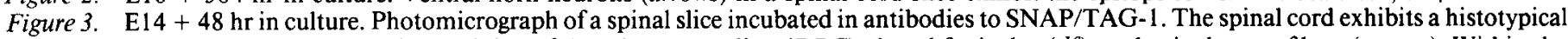

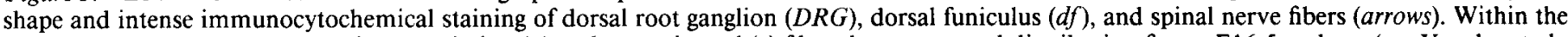

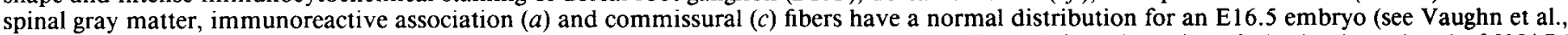

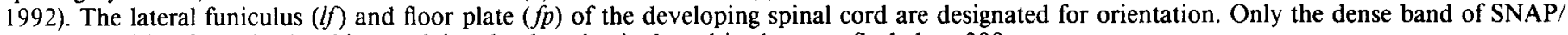
TAG-1-positive fibers in the skin overlying the dorsal spinal cord is aberrant. Scale bar, $300 \mu \mathrm{m}$.


(arrowheads) in a spinal cord slice. Scale bar, $30 \mu \mathrm{m}$. 

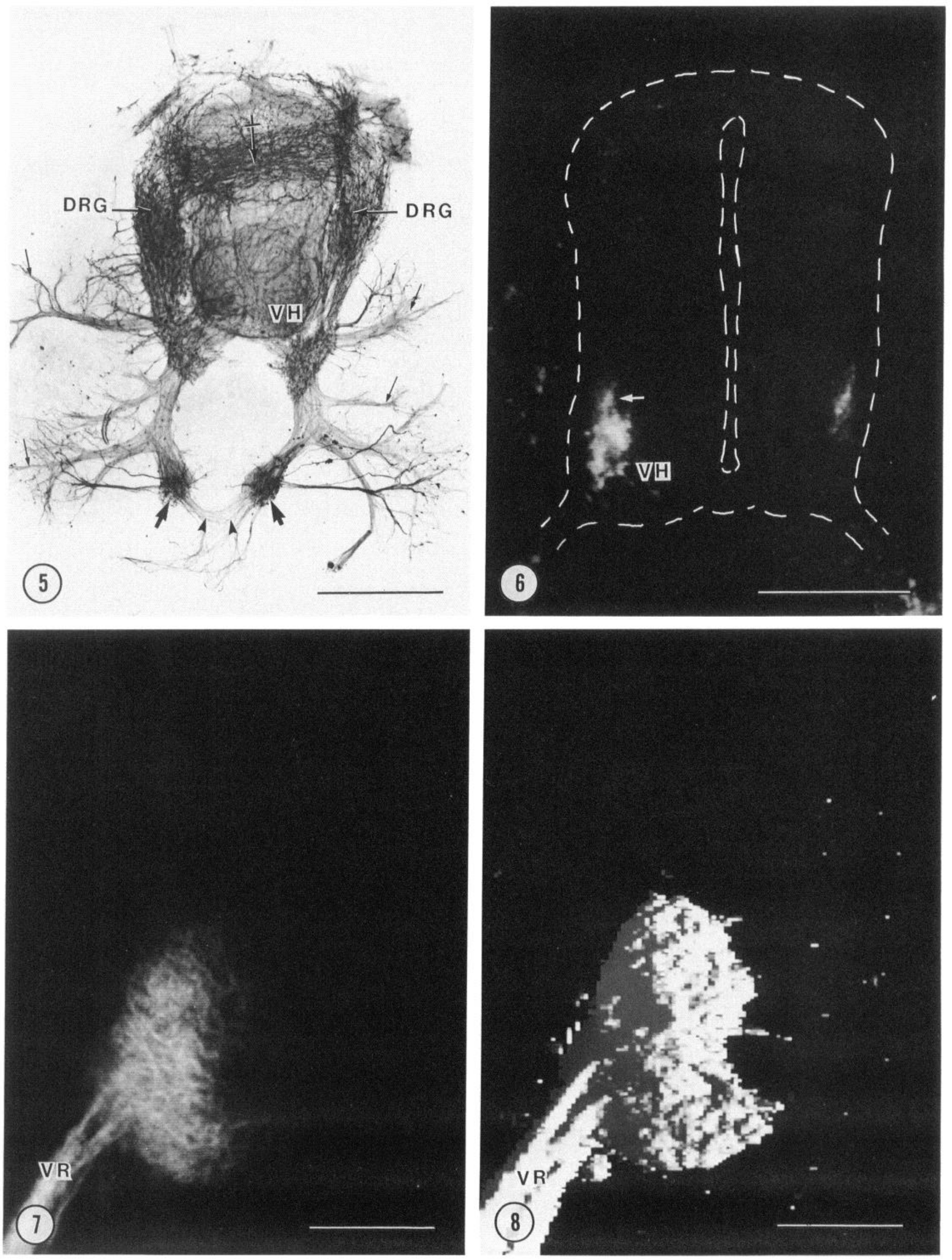

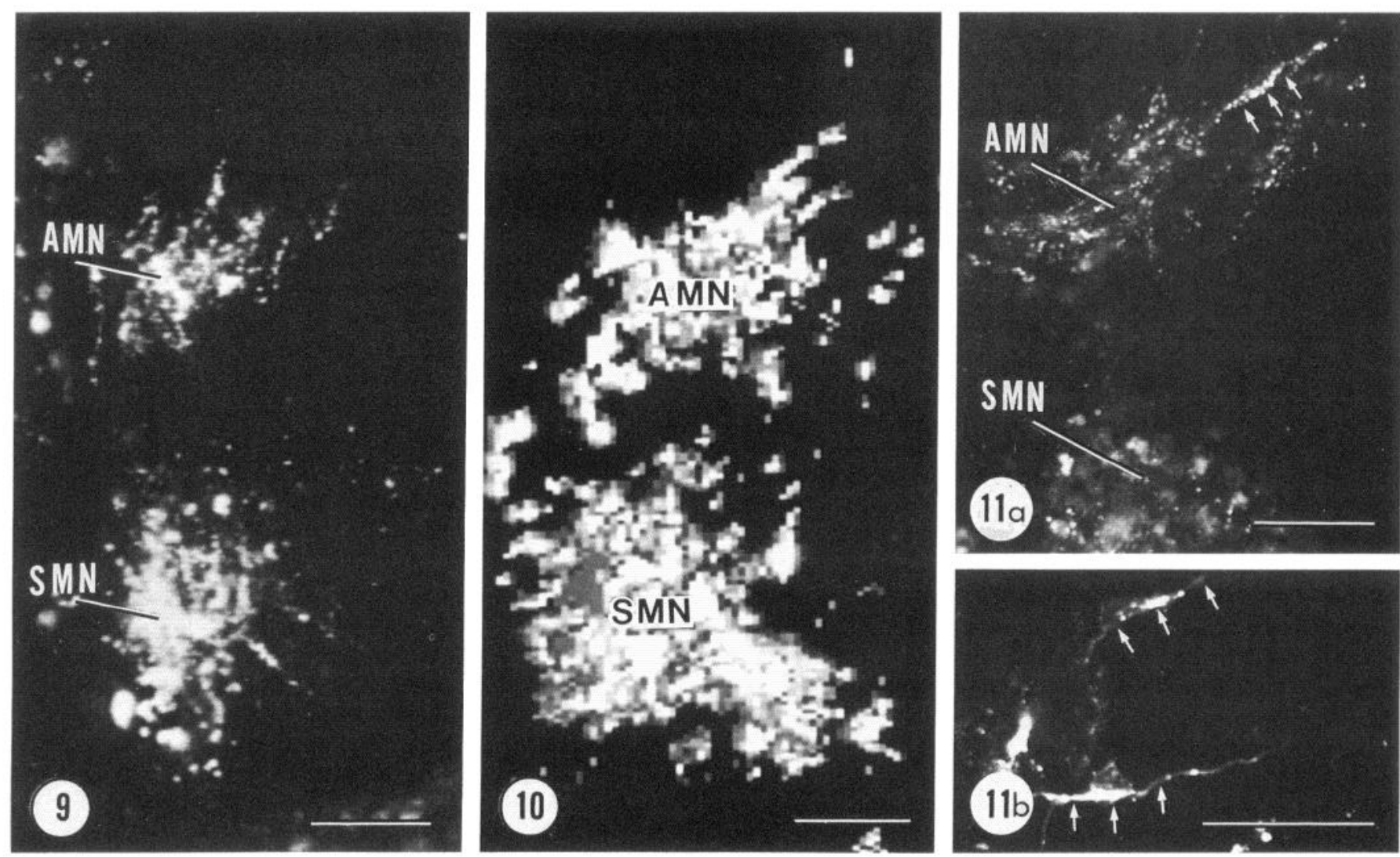

Figure 9. E14 $+72 \mathrm{hr}$ in culture. DiI injection of living specimen. Confocal microscopy of a single optical slice shows the separation of motor neurons into distinct, autonomic motor neuron $(A M N)$ and somatic motor neuron $(S M N)$ subcolumns in the intermediate and ventral spinal cord, respectively.

Figure 10. E14 $+72 \mathrm{hr}$ in culture. Dil injection of living specimen. A three-dimensional reconstruction of the specimen in Figure 9 confirms that AMNs are separated from the SMNs throughout the entire thickness of the slice.

Figure 11. E14 $+72 \mathrm{hr}$ in culture. DiI injections of living specimens. During dorsal translocation into the presumptive intermediolateral horn, some AMNs appear to move medially, presumably to form the intercalated and central AMN nuclei. The slice preparation in $a$ demonstrates the separation of the AMN and SMN subcolumns by $72 \mathrm{hr}$ in culture, and also shows a mediolaterally oriented AMN neuron (arrows); $b$ shows a higher-magnification image of other medially directed AMNs (arrows) from another spinal slice. Dorsal is up and medial is to the right in Figures 9-11. Scale bars, $50 \mu \mathrm{m}$.

same slice was observed daily, we could monitor the progression of motor neuronal development from a single, primitive motor column into two fully separated subcolumns.

\section{Nature of $A M N$ translocation}

In this and previous studies (Markham and Vaughn, 1991; Phelps et al., 1991a), care has been taken to designate our observations as neuronal translocations rather than migrations because, pres- ently, there is no direct evidence that AMNs are actively migrating. Despite this lack, cellular migration is the most likely interpretation for AMN translocation if two alternative possibilities can be excluded. The first possibility is that the dorsally located AMNs may be a separate, more dorsally located cell population from the beginning, and that they only appear to translocate dorsally because they become labeled by retrograde tracer at older embryonic stages due to a later arrival of their

Figure 5. E14 + $24 \mathrm{hr}$ in culture. A whole-mount spinal slice that was stained immunocytochemically for neurofilament protein. Motor projections from the ventral horn $(V H)$ into regions containing somatic muscles (small arrows; muscles not stained) and paravertebral sympathetic ganglia (large arrows) appear histotypic. Robust dorsal root ganglia $(D R G)$ and primary afferent fibers are present, and the specimen exhibits a histotypic appearance except for aberrant fibers that project superficially across the dorsal horn (crossed arrow) and those that form a small commissure between the sympathetic ganglia (arrowheads). Scale bar, $500 \mu \mathrm{m}$.

Figure 6. E14 $+24 \mathrm{hr}$ in culture. Target areas of SMN and AMN projections were injected with DiI (see Materials and Methods) shortly after explanting the spinal slice. Dye uptake in the ventral horn $(V H)$ of the living slice was then recorded with a confocal microscope. Most of the dye was associated with ventral horn cells in a single, primitive motor column, but a few cells (arrow) appeared to have begun a dorsal translocation. Only the dorsalmost cells of the primitive motor column (putative AMNs) on the right side of the spinal cord are apparent in this single optical section. For orientation, the outline of the entire transversely sectioned spinal cord has been traced from a transmitted light image of the slice preparation. Scale bar, $250 \mu \mathrm{m}$.

Figure 7. E14 $+2 \mathrm{hr}$ in culture. Digitized images of single spinal slice that was fixed prior to injection with DiI. A single, confocal optical section reveals AMNs and SMNs clustered in a single, tightly packed primitive motor column. Exiting motor axons form the ventral root ( $V R)$.

Figure 8. E14 $+2 \mathrm{hr}$ in culture. Digitized images of single spinal slice that was fixed prior to injection with DiI. A three-dimensional reconstruction of 22 consecutive, $10-\mu$ m-thick optical sections of the same spinal slice confirms that the primitive motor column is a single, compact group of cells throughout the entire thickness of the slice. Scale bar, $100 \mu \mathrm{m}$. 

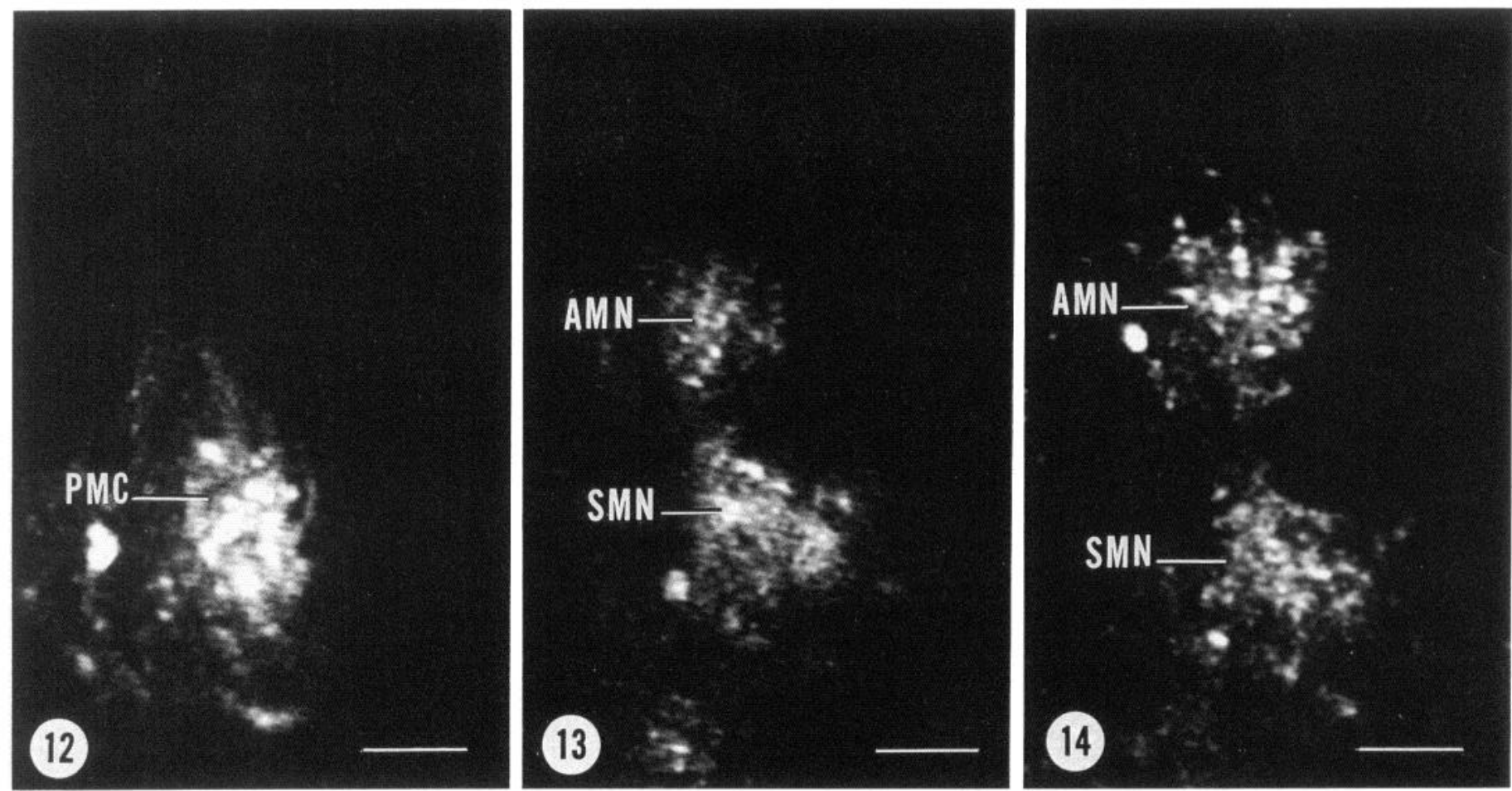

Figure 12. E14 $+24 \mathrm{hr}$ in culture. DiI injection of a living specimen. Figures 12-14 are three confocal micrographs of the same spinal cord slice that was photographed every $24 \mathrm{hr}$. Together, these sequential images from the same living slice illustrate the daily progression in vitro of the separation of AMNs and SMNs. In Figure 12, there is a single, ventrally located primitive motor column $(P M C)$ containing both AMNs and SMNs. The lightly fluorescent staining at the top of the primitive motor column probably represents a few cells that have just begun their dorsal translocation. Figure 13. E14 + $48 \mathrm{hr}$ in culture. The dorsoventral separation of AMNs and SMNs is well under way.

Figure 14. E14 $+72 \mathrm{hr}$ in culture. The dorsoventral separation of AMNs and SMNs is pronounced with approximately equal numbers of cells comprising the two motor subcolumns. Scale bars, $50 \mu \mathrm{m}$.

axons within the tracer-injected paravertebral ganglia. However, a number of observations argue against this theoretical possibility. For example, an identical translocation pattern to that obtained by HRP retrograde labeling from autonomic ganglia (Markham and Vaughn, 1991) is revealed by the technique of ChAT immunocytochemistry (Phelps et al., 1991a). Since the expression of ChAT immunoreactivity within spinal motor neurons is known to precede the time that the axons of these cells reach their peripheral targets (Rubin, 1985a,b; Phelps et al., 1990, 1991a), it should provide an independent measure of AMN translocations. Thus, the same dorsal progression of both ChAT-positive and HRP-labeled AMNs argues against the idea that a delayed visualization of a later-forming, more dorsally located population of AMN precursors was the actual basis for the apparent dorsal translocation of these cells. In addition, as reported in the present study, a persuasive argument against a delayed differentiation of dorsally located AMN precursors is provided by the fact that dorsal translocations of AMNs oc- curred in cultured spinal slice preparations even when the DiI injected paravertebral ganglia were removed from the cultured slices. After this microsurgical manipulation, the ganglia could no longer have served as sources of retrograde label for laterarriving axons. Consequently, DiI-labeled AMNs in the intermediolateral column following $72 \mathrm{hr}$ in culture must have been labeled when they all were still located within the ventral, primitive motor column (E14, onset of culture period).

Since the available evidence strongly favors a dorsal translocation of AMNs from the primitive motor column, the question arises as to whether this is due to a passive, dorsal displacement of AMNs by the insertion of either newly generated cells or neuropil between the two motor neuronal subsets. Available observations cast doubt upon this possibility. First, it seems clear that the transition from the mixture of AMNs and SMNs in the E13.5 primitive motor column to the dorsoventral segregation observed at E14-E14.5 (Markham and Vaughn, 1991) would be difficult to achieve by a simple, passive displacement

Figure 15. E14 + 72 hr in culture. In Figures 15-17, the autonomic ganglia and ventral roots of spinal slices were injected with DiI either following fixation (Figs. 15, 16) or in the living state (Fig. 17). In Figure 15, a motor neuron (arrows) in an intermediate position between the ventral horn $(V H)$ and the intermediolateral gray $(I M L)$ displays a bipolar, dorsoventral alignment. In addition, an AMN in the IML (arrowheads) exhibits a mediolateral orientation. The ventral root $(V R)$ is shown for orientation. Scale bar, $50 \mu \mathrm{m}$.

Figure 16. E14 $+72 \mathrm{hr}$ in culture. A higher magnification of the IML illustrates a dorsally located AMN (arrowheads) that is mediolaterally aligned, as well as a more medially positioned AMN (arrows) that displays an orientation that is intermediate to both the dorsoventral and mediolateral axes of the spinal cord. Dorsal is up and medial is to the right in both Figures 15 and 16. Scale bar, $50 \mu \mathrm{m}$.

Figure 17. E14 $+72 \mathrm{hr}$ in culture. Four hours following DiI injection of the autonomic chain ganglia and ventral roots of a living slice, all injection sites were removed microsurgically from the explant. Confocal micrograph of the slice shows that, despite the absence of their peripheral targets, AMNs have separated dorsally from the SMNs to form distinct subcolumns on both sides of the spinal cord. Scale bar, $100 \mu \mathrm{m}$. 

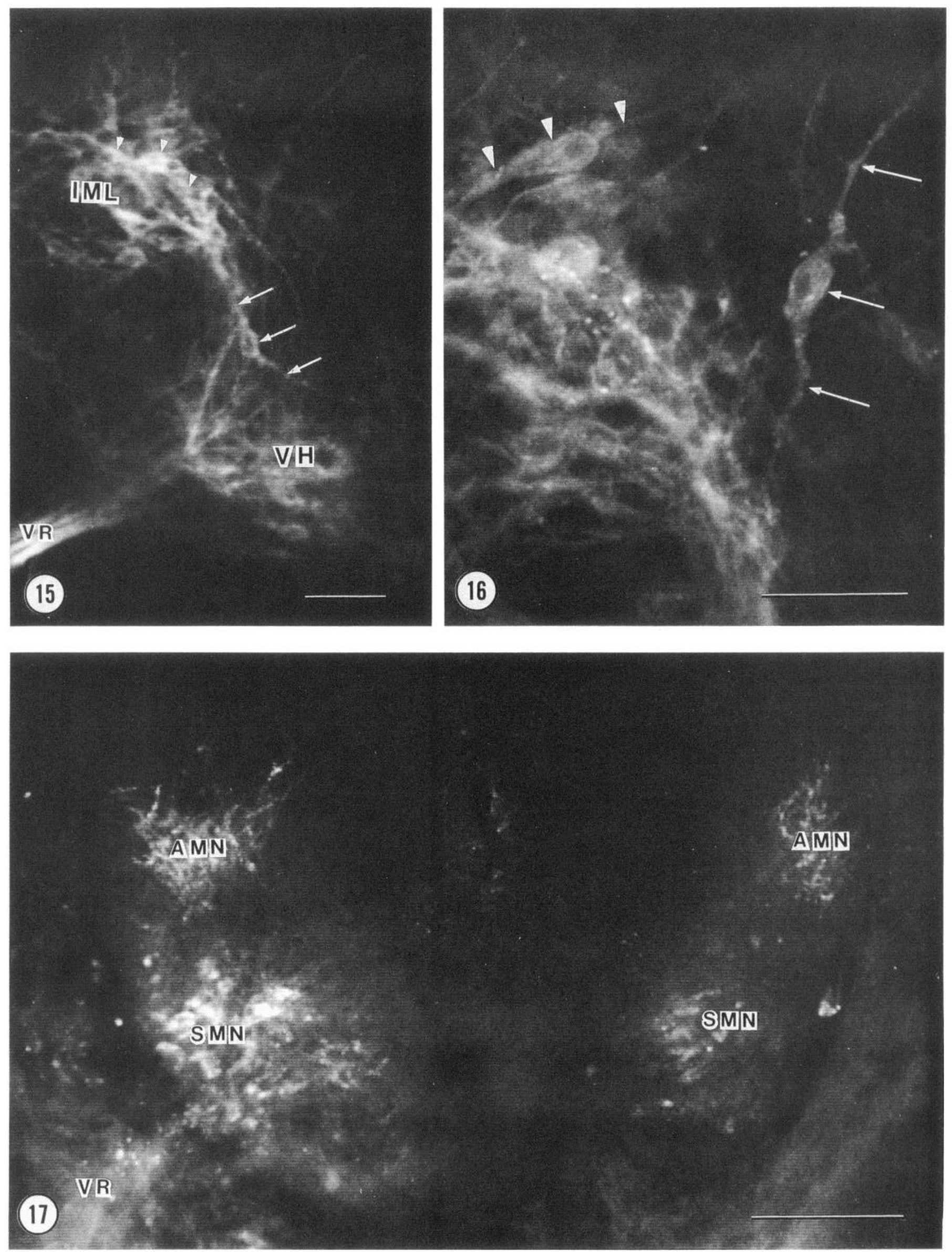


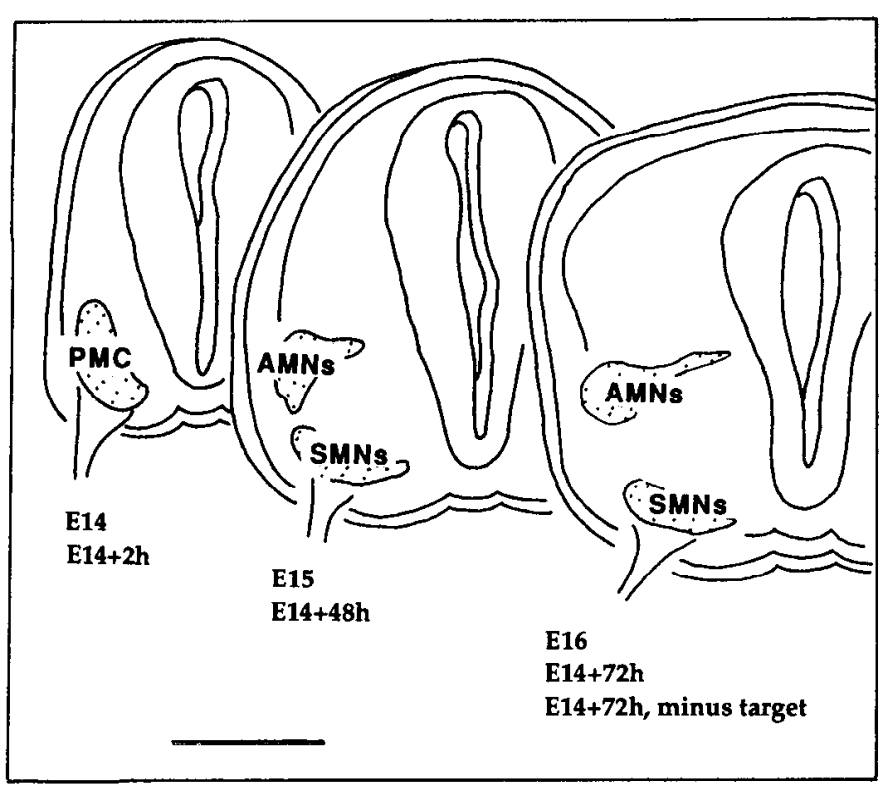

Figure 18. Schematic summary of motor neuronal migratory patterns. At $\mathrm{E} 14$ in vivo and $\mathrm{E} 14+2 \mathrm{hr}$ in vitro, $\mathrm{AMNs}$ and SMNs are closely packed within a common primitive motor column $(P M C)$; by $\mathrm{E} 15$ in vivo and E14 + $48 \mathrm{hr}$ in vitro, many AMNs have separated substantially dorsally from the SMNs, and the stage represented by E16 in vivo and E14 $+72 \mathrm{hr}$ in vitro shows a complete separation of these two subsets of motor neurons. This separation also takes place in spccimens wherc the peripheral targets of AMNs have been removed microsurgically from the slices $(E 14+72 \mathrm{hr}$, minus target $)$. Medially directed translocations of AMNs are also seen in the latter two stages. Scale bar, $250 \mu \mathrm{m}$.

caused by an ingrowth of new cells or the expansion of developing neuropil. Both mechanisms would appear to lack the precision necessary to sort out and systematically displace the two, intermixed subsets of motor neurons. Thus, the initial conversion from an integrated distribution to a dorsoventral segregation of AMNs and SMNs within the primitive motor column implies an active cellular migration. Another argument against a passive displacement of AMNs is that translocating cells exhibited the same morphological features as actively migrating neurons in other regions of the developing CNS (e.g., Rakic, 1972, 1990). During the period of the dorsal translocation, AMNs are bipolar cells whose processes are directed in the axis of cellular movement (Markham and Vaughn, 1991; Phelps et al., 1991a). A third argument against the passive displacement hypothesis is that observations made at closely spaced, intermediate times revealed that the apparent movements of AMNs were consistent with progressive transitions that started with a few cells and continued with increasingly more numerous AMNs becoming separated dorsally from the SMNs in the ventral spinal cord. This is in contrast to the pattern that might be expected if a single, large group of cells was being split into two subunits by the insertion of other tissue components.

Thus, presently available evidence does not favor the possibilities either that AMNs arise from a separate, later-forming subpopulation of cells located dorsally to the SMNs, or that they are passively displaced from the primitive motor column by the insertion of other histogenic elements. Therefore, the possibility of an active migration of AMNs is a logical, third alternative. If AMNs are actively migrating, the question arises as to what elements of the developing spinal cord might serve as guides or substrates for this migration. The most commonly defined pathway for neuronal migration is provided by radial glial processes (Rakic, 1972, 1990; Hatten, 1990; Hatten and Mason, 1990), but, in the case of AMNs, the orientation of such radial processes is perpendicular to the direction of cellular movement (Markham and Vaughn, 1991; Phelps et al., 1991a). In contrast, the axons of early-forming circumferential spinal interneurons exhibit the appropriate spatial and temporal characteristics to provide a route for the AMN migration (Phelps et al., 1991b, 1993; Vaughn et al., 1992). This possibility is based on circumstantial evidence and requires experimental validation. The present findings provide an important step in this direction by showing that AMN translocation patterns in vivo are mimicked in the cultured spinal slice preparation, thereby allowing for such experimental perturbations of AMN migrations within this his totypic, yet accessible, developing system.

\section{Relation of cellular translocations to peripheral target}

The utility of the spinal slice preparation for such experimental manipulations has been demonstrated in the present investigation by the microsurgical removal of the synaptic targets of AMNs in the periphery. The fact that both AMNs and SMNs have their axons in close proximity to their respective peripheral synaptic targets while they are still intermixed in the primitive motor column (Markham and Vaughn, 1991) suggests the possibility that some differentiation factor derived from the periphery might play a role in the formation of separate motor neuronal subcolumns. Specifically, one might suspect that AMN migration depends upon some signal(s) derived from the paravertebral chain ganglia. The spinal slice provided a means to test this possibility since the effect of target removal on subsequent $A M N$ translocation could be ascertained. These experiments showed that continuous axonal connection to synaptic targets was not necessary to sustain the AMN translocations that occur in developing rat spinal cord. However, since AMNs were identified by retrograde labeling from the peripheral targets, the present experiments did not address the issue of whether initial contacts of AMN axons with their peripheral targets might have been sufficient for a one time induction in AMNs that affected their subsequent migration. Evidence against this possibility is available from studies conducted in chick embryos (e.g., Prasad and Hollyday, 1991), but it remains to be obtained for the more complex AMN migration patterns of mammalian species.

\section{Conclusions}

The findings of this investigation (1) have shown that in vivo translocation patterns of motor neurons are mimicked within in vitro, organotypic spinal slices (Fig. 18); (2) have confirmed the dorsal translocation of autonomic motor neurons; (3) have strengthened the possibility that this translocation is due to an actual cellular migration; (4) have shown that the dorsal and dorsomedial movements of autonomic motor neurons are independent of continuous connection with peripheral targets (Fig. 18); and (5) have demonstrated the utility of embryonic, mammalian spinal slices for experimental analyses designed to define the structural and molecular substrates of motor neuronal migration. Once such substrates have been defined, it will become feasible to examine the possible roles played by migratory pathways upon the differentiation of motor neuronal phenotypes. 


\section{References}

Barber RP, Phelps PE, Vaughn JE (1991) Generation patterns of immunocytochemically identified cholinergic neurons at autonomic levels of the rat spinal cord. J Comp Neurol 311:509-519.

Braschler UF, Iannone A, Spenger C, Streit J, Luscher HR (1989) A modified roller tube technique for organotypic cocultures of embryonic rat spinal cord, sensory ganglia and skeletal muscle. J Neurosci Methods 29:121-129.

Bronner-Fraser M, Fraser SE (1988) Cell lineage analysis reveals multipotency of some avian neural crest cells. Nature 335:161-164.

Chen EW, Chiu AY (1992) Early stages in the development of spinal motor neurons. J Comp Neurol 320:291-303.

Dodd J, Morton SB, Karagoeos D, Yamamoto M, Jessell TM (1988) Spatial regulation of axonal glycoprotein expression on subsets of embryonic spinal neurons. Neuron 1:105-116.

Freshney RI (1987) Culture of animals cells-a manual of basic techniques, p 346. New York: Wiley.

Gähwiler BH (1981) Organotypic monolayer cultures of nervous tissue. J Neurosci Methods 4:329-342.

Gähwiler BH, Rietschin L, Knopfel T, Enz A (1990) Continuous presence of nerve growth factor is required for maintenance of cholinergic septal neurons in organotypic slice cultures. Neuroscience 36:27-31.

Hatten ME (1990) Riding the glial monorail-a common mechanism for glial-guided neuronal migration in different regions of the developing mammalian brain. Trends Neurosci 13:179-184.

Hatten ME, Mason CA (1990) Mechanisms of glial-guided neuronal migration in vitro and in vivo. Experientia 46:907-916.

Honig MG, Hume RI (1989) Versatile fluorescent dyes for neuronal labeling and pathway tracing. Trends Neurosci 12:333-341.

Leber SM, Breedlove SM, Sanes JR(1990) Lineage, arrangement, and death of clonally related motoneurons in chick spinal cord. J Neurosci 10:2451-2462.
Markham JA, Vaughn JE (1991) Migration patterns of sympathetic preganglionic neurons in embryonic rat spinal cord. J Neurobiol 22: 811-822.

Phelps PE, Barber RP, Brennan LA, Maines VM, Salvaterra PM, Vaughn JE (1990) Embryonic development of 4 different subsets of cholinergic neurons in rat cervical spinal cord. J Comp Neurol 291:9-26.

Phelps PE, Barber RP, Vaughn JE (1991a) Embryonic development of choline acetyltransferase in thoracic spinal motor neurons. Somatic and autonomic neurons may be derived from a common cellular group. J Comp Neurol 307:77-86.

Phelps PE, Barber RP, Vaughn JE (1991b) Substrates underlying the migratory patterns of sympathetic preganglionic neurons in embryonic rat. Soc Neurosci Abstr 17:206.

Phelps PE, Barber RP, Vaughn JE (1993) Embryonic development of rat sympathetic preganglionic ncurons: possible migratory substrates. J Comp Neurol 330:1-14.

Prasad A, Hollyday M (1991) Development and migration of avian sympathetic preganglionic neurons. J Comp Neurol 307:237-258.

Rakic P (1972) Mode of cell migration to the superficial layers of fetal monkey neocortex. J Comp Neurol 145:61-84.

Rakic P (1990) Principles of neural cell migration. Experientia 46: 882-891.

Rubin E (1985a) Development of the rat superior cervical ganglion: ingrowth of preganglionic axons. J Neurosci 5:685-696.

Rubin E (1985b) Development of the rat superior cervical ganglion: initial stages of synapse formation. J Neurosci 5:697-704.

Vaughn JE, Phelps PE, Yamamoto M, Barber RP (1992) Association interneurons of embryonic rat spinal cord transiently express the cell surface glycoprotcin SNAP/TAG-1. Dev Dynam 194:43-51.

Yamamoto M, Boyer AM, Crandall JE, Edwards M, Tanaka H (1986) Distribution of stage-specific neurite-associated proteins in the developing murine nervous system recognized by a monoclonal antibody. J Neurosci 6:3576-3594. 